\title{
Dynamic effects in plasma wakefield excitation
}

\author{
R. G. Hemker and W. B. Mori \\ Department of Physics and Astronomy, University of California at Los Angeles, Los Angeles, California 90095-1547
}

S. Lee and T. Katsouleas

Department of Electrical Engineering, University of Southern California, Los Angeles, California 90089-0271

(Received 2 December 1999; published 26 June 2000)

\begin{abstract}
We have used 2D cylindrically symmetric particle-in-cell simulations to investigate the dynamics of a high energy electron beam propagating through an underdense plasma. The simulation parameters are relevant to a recent plasma wakefield experiment conducted at the Stanford Linear Accelerator Center [R. Assmann et al., Stanford Linear Accelerator Center Proposal, 1997]. We model the dynamic development of the beam and wakefield excitation over meters of propagation length. To most clearly illustrate the dynamics of both the beam and the wakefield, a video of the simulation data is presented. The main observation is that the beam dynamics, i.e., its betatron motion in the resulting ion channel, agree well with the theoretical predictions while the plasma wake remains almost invariant over the entire propagation distance. The video illustrates subtle details regarding the interplay between the beam dynamics and wakefield generation. The results presented here complement results published separately [S. Lee et al., Phys. Rev. E 61, 7014 (2000)].
\end{abstract}

PACS numbers: 52.65.Rr, 52.40.Mj, 41.75.-i

\section{INTRODUCTION}

The basic concept of a plasma wakefield accelerator (PWFA) is to accelerate a low current trailing electron bunch by the wakefield generated by a high current driver $[1,2]$. If the driving bunch is highly relativistic, then both the accelerating as well as the accelerated bunch are moving with about the speed of light and the accelerated bunch can stay in phase with the accelerating field for distances long enough to gain significant amounts of energy. Motivated by and as part of the preparations for an experiment which was recently conducted at the Stanford Linear Accelerator Center (SLAC), we have simulated a plasma wakefield accelerator with the expected parameters of this experiment [3]. In this experiment a $30 \mathrm{GeV}$ electron beam at SLAC is used to excite a wake of the order of $1 \mathrm{GeV} / \mathrm{m}$ in a $1.4 \mathrm{~m}$ long plasma of density $1-2 \times 10^{14} \mathrm{~cm}^{-3}$. In this wake the centroid energy of the tail of the beam is expected to increase by several hundred MeV. Since the beam in this experiment is typically much denser than the plasma (e.g., $N=3.5-4 \times 10^{10}$ electrons in a $\sigma_{z}=0.6 \mathrm{~mm}$ bunch length and a spot size of $\sigma_{r}=50 \mu \mathrm{m}$ corresponding to a beam density $n_{b}=1 \times 10^{15} \mathrm{~cm}^{-3}$ ), the PWFA is in the highly nonlinear or so-called blowout regime [4]. The advantages that this regime offers are a high accelerating gradient, a constant accelerating structure with respect to the transverse dimensions, a linear focusing force, and a high transformer ratio. However, in this nonlinear regime, linear theory or fluid models are inapplicable and do not provide an accurate understanding of the physics. Much better insight into the physical processes can be gained by using particle-in-cell (PIC) simulations, which allow ac- curate modeling of highly nonlinear processes such as the ones occurring here. For these reasons, we conducted PIC simulations to investigate this regime of plasma wakefield acceleration. Many of these results can be found elsewhere [5]. In this paper we provide more detail on the dynamics of the drive beam and wakefield evolution. The presentation is aided through simulation generated videos.

\section{SIMULATION SETUP AND RESULTS}

We carried out simulations for the physical parameters similar to the ones described above using the newly developed, object-oriented, parallel PIC code OSIRIS [6]. This PIC code is fully explicit and therefore contains all of the relevant physics. Because of its object-oriented structure, this code contains algorithms for several geometries and dimensions as well as current deposition schemes. The algorithms for the results presented in this paper were $2 \mathrm{D}$ cylindrically symmetric, and a rigorously charge conserving current-deposition algorithm was used [7]. The code also uses a moving simulation window [8], which moves at the speed of light, i.e., with the beam. This limits the simulation domain to the beam and its immediate surroundings rather than the whole propagation distance of the beam. The simulation window in normalized units had a size along the propagation direction $z$ of $25 c / \omega_{p}$ and a size in the radial direction $r$ of $10 c / \omega_{p}$ with a grid of $N_{z} \times N_{r}=500 \times 200$. Here $c$ is the speed of light and $\omega_{p}$ is the plasma frequency for a given plasma density $n_{p}$. We will use $n_{p}=2.1 \times 10^{14} \mathrm{~cm}^{-3}$, which corresponds to $c / \omega_{p}=0.367 \mathrm{~mm}$, throughout this paper when converting simulation results back into physical units. 
This means the simulation window corresponds to a size of $9.175 \mathrm{~mm} \times 3.67 \mathrm{~mm}$. The beam propagated through the plasma for 190000 time steps with $d t=0.02 \omega_{p}^{-1}$ (corresponding to $18.35 \mu \mathrm{m}$ of propagation distance per time step) for a total of $3800 c / \omega_{p}(\sim 1.4 \mathrm{~m})$. Nine particles per cell were used for the background plasma and 25 particles per cell for the beam. The beam's longitudinal profile was fitted to the experimentally known profile of the SLAC beam [3], which is very close to a Gaussian profile of width $\sigma_{z}=0.63 \mathrm{~mm}$, and transversely as a Gaussian of width $\sigma_{r}=70 \mu \mathrm{m}$. The peak density was taken to be $7.56 \times 10^{14} \mathrm{~cm}^{-3}$. Therefore the simulated electron bunch contained $3.7 \times 10^{10}$ electrons.

Figures 1(a) and 1(b) show the first and the last frames of the video accompanying this paper. The video shows seven different aspects of the simulation data. In the upper left-hand corner is a colored, rubber sheet representation of the longitudinal, accelerating electric field. For this visualization the elevation of a surface point as well as its color represent the field strengths of the electric field. Note that we chose a perspective for visualizing the rubber sheet surface so that negative field values of the electric field would be represented by positive values of the surface elevation. This leads to a better visualization of the accelerating region. The sharp edge of the rubber sheet surface going roughly from the upper lefthand corner to the lower right-hand corner is due to the axial boundary of the 2D cylindrically symmetric simulation and, accordingly, $r$ increases starting from this axial boundary toward the lower left-hand corner. Because of the chosen perspective, the rubber sheet does not show the data for the whole simulation. The upper middle of the video frame shows the value of the longitudinal electric field along the axial boundary for its full length of $25 c / \omega_{p}$. The figure in the lower middle below the electric field lineout shows the energy gain and loss of the
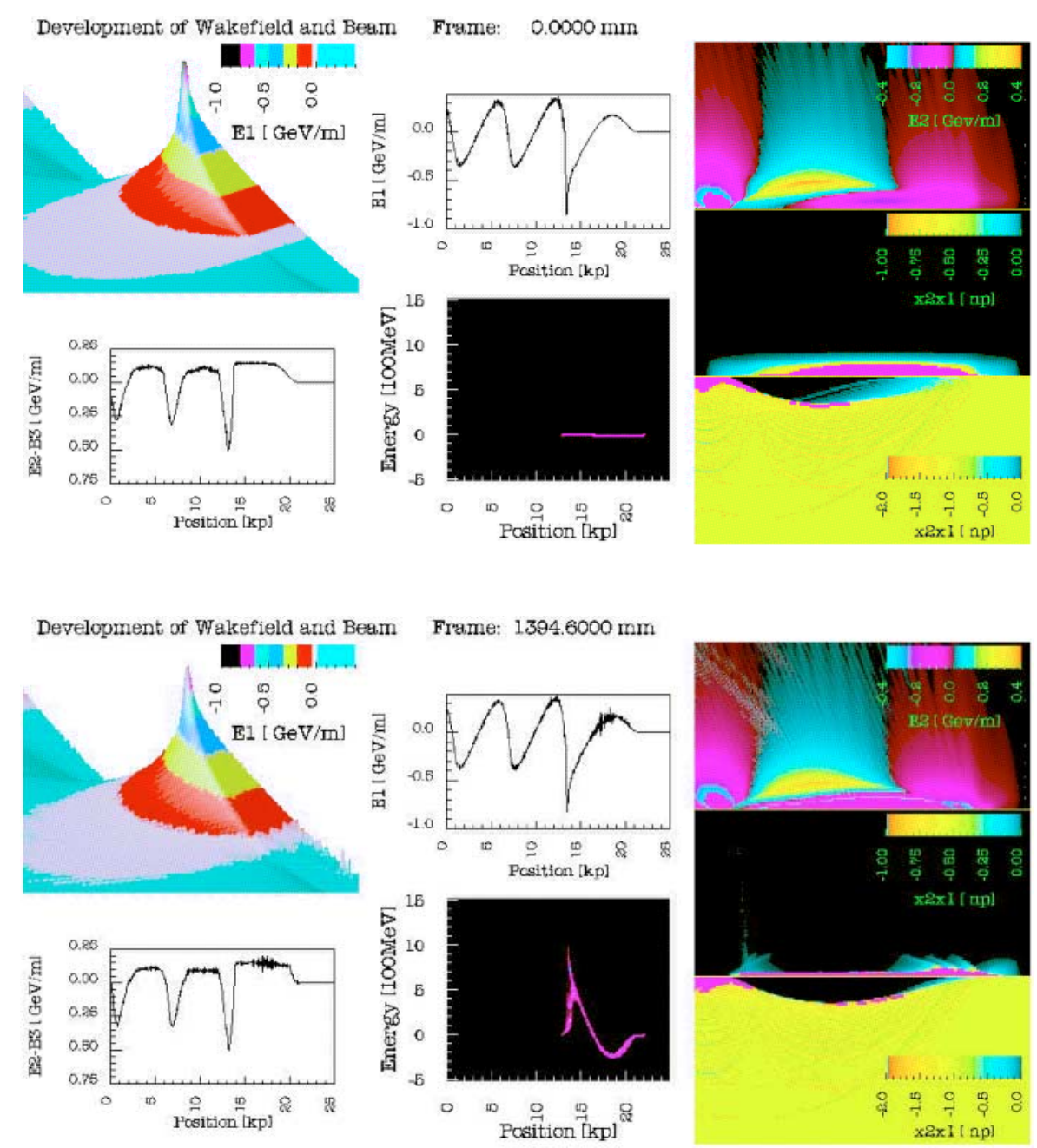

FIG. 1. Video The first and the last frames of the vided accompanying this paper. The different plots of the video are (column by column from the top to the bottom) $E_{z}$ : rubber sheet; $E_{r}-B_{\theta}$ : focusing field lineout $\sigma_{r}$ off axis; $E_{z}$ : lineout on axis, beam-energy change as a function of $z-c t ; E_{r}$ in the $r-z$ plane; $n_{\text {beam }}$ in the $r-z$ plane; $n_{\text {plasma }}$ in the $r-z$ plane (direction of $r$ reversed). Please note that in the figures and the video the indices 1,2 , and 3 are used instead of $z, r$, and $\theta$. 
electron beam as a function of the axial position. The colored areas indicate the parts of this plot where beam electrons are present. Note that the horizontal axis of this plot is precisely aligned with the axis of the field lineout above. The plot in the lower left-hand corner shows the focusing field experienced by the beam electrons, $E_{r}-B_{\theta}$, at a position $73.4 \mu \mathrm{m} \cong \sigma_{r}$ off axis. The right-hand column shows three color plots in the $r-z$ plane (in the video frame also labeled as the $x 2-x 1$ plane). The plots shown (from top to bottom) are the radial electric field $E_{r}$, the charge density of the beam, and the charge density of the background plasma. This last plot has been mirrored along the axis to allow for a more direct comparison of the plasma density with the beam density. The horizontal axis for each of the three plots goes from 12.5 to $22.5 c / \omega_{p}$ of the simulation window and the vertical axis shows 0 to $5 c / \omega_{p}$ along the radial direction. The field and density values are given by the color bars in each of the plots. Note that the areas of the plots colored in magenta are areas in which the field or density values are outside the respective color scales. Since the color code of the beam charge density plot reaches from 0 to 1 , which is the normalized density of the background plasma, the magenta-colored areas in this plot indicate densities above the background plasma density.

The video consists of a total of 191 frames at a speed of 10 frames/sec. With such a large number of frames the video can clearly illustrate subtle points in the evolution of the beam and wake. The first fact to note when watching the video is the lack of change over time in the evolution of the accelerating electric field and the focusing field. With the exception of the peak accelerating field, which fluctuates slightly by about $\pm 0.05 \mathrm{GeV} / \mathrm{m}$ around a value of about $0.75 \mathrm{GeV} / \mathrm{m}$ (about $\pm 7 \%$ ), and some slight variation in the level of numerical noise, the accelerating electric field essentially does not change over time. This is in strong contrast to the dynamic development of the beam radius (middle plot in the right-hand column) and energy (lower plot in the center column) and the radial electric field (upper plot in the right-hand column). The energy plot shows that every part of the beam except the front part and the very tail gains or loses energy linearly as a function of time. This is consistent with the constant longitudinal field since at an initial energy of about $30 \mathrm{GeV}$ the beam electrons experience no significant phase slippage over the time of the simulation.

Two other effects can also be observed. First, there is a slight broadening of the front part of the decelerated area of the beam along the energy axis, which means that not all electrons at a given $z$ experience exactly the same decelerating field. Second, there is a large energy spread of the very back of the beam tail, which splits into two parts. The first observation can be understood when looking at the background plasma charge density. The plasma charge density plot shows that in the front part of the beam the area of total electron blowout is smaller than in the latter parts of the beam, and therefore the radius up to which the focusing force $F_{r}$ is independent of $z$ is smaller. According to the Panofsky-Wenzel theorem, $\partial F_{r} / \partial z=\partial F_{z} / \partial r$, this implies an acceleration gradient that varies along the radial position [4]. This can also be noticed for the region of decelerating field that is visible in the lower right-hand corner of the $E_{z}$ rubber sheet plot. The radially flat area increases slightly in width toward the back. The broadening of the front part of the deceleration area of the beam is a result of this nonuniform accelerating field. The energy spread of the tail of the beam can be understood by looking at the narrowing of the accelerating and focusing field profile near the peak accelerating field. It shows that a part of the tail of the beam, in contrast to the rest of the beam, experiences a strong defocusing force that pushes it radially out of the accelerating field. The blowout of some of the tail electrons of the beam can also be seen in the development of the beam charge density.

The evolution of the main part of the beam, as seen in the beam charge density plot, is clearly dominated by the betatron oscillation of the beam in the focusing field. The focusing field is mainly due to the ions left in the plasma blowout area, as seen in the plasma charge density plot, since the effects of electric and magnetic fields of the relativistic beam on itself cancel each other almost completely. The linear focusing force in the blowout area results in the same oscillation frequency for all beam electrons in that area. The beam propagates while undergoing betatron oscillations with a wavelength of the spot size

$$
\lambda_{\text {spot size }}=\lambda_{\beta} / 2=\pi \sqrt{\frac{\gamma m c^{2}}{2 \pi e^{2} n_{0}}},
$$

where $\lambda_{\beta}$ is the betatron wavelength of a single particle. Measuring this wavelength using the minima of the oscillation of the beam density gives a wavelength $\lambda_{\beta} / 2=40 \mathrm{~cm}$ as predicted by Eq. (1) for the density of the simulation [3].

The dynamics of the front part of the beam is more complex because the blowout area there is not as wide. This leads to nonharmonic oscillations or so-called aberrations in the focusing force, which leads to phase mixing of the electrons. The oscillation frequency of the beam electrons decreases toward the front. The video shows clearly that after the main part of the beam reaches an oscillation minimum this minimum moves forward toward the front of the beam as the electrons there execute betatron oscillations with lower frequencies. This dynamics at the front of the beam leads to a subtle point, which was not appreciated until the video was made. Namely, the focusing field for the beam, $E_{r}-B_{\theta}$, shows an unexpected behavior with time. Initially the focusing force rises slowly over the first one-fourth of the beam, but once the head of the beam begins to pinch the rise becomes steeper. The 
unexpected behavior results because the transverse profile never relaxes back to the original one. Instead, there is always an axial slice of the beam at the head of the beam that is near a pinch. So on average, the beam density at the front of the beam is always larger than it was at $t=0$. As a result, the occurrence of complete blowout is earlier in the beam and the region of blowout is wider leading to more of the beam undergoing the uniform betatron oscillations than might have been expected.

Unlike the beam, the plasma electrons respond predominantly to only $E_{r}$. Thus, the blowout of the plasma electrons and their oscillation back onto the axis in the back of the pulse is caused by the total radial electric field that they experience. The video shows that the radial field has two distinct regions. The front, where the plasma electrons are not blown out yet, is dominated by the electric field of the beam; the back, where the plasma electrons are blown out, is dominated by the radial electric field of the remaining ion charge. The plasma charge density plot shows the effect of this. In the moving window frame, i.e., in the $z$-ct coordinate, the plasma electrons stream backward past the stationary drive beam. After the radial field force deflects the electrons outward, most of them coalesce in a narrow, high density surface layer that lies at the edge of the blowout region. The radius of the blowout region [5], and therefore the radial position of the layer, is roughly $2 \sigma_{r} \sqrt{n_{b} / n_{0}}$. The electrons stream backward within this narrow surface layer and converge on the axis creating a very dense spike and, therefore, a sharp peak in the accelerating field. (Note that in the laboratory frame individual electrons are blown out and then return while remaining near their initial $z$ value, but we will use the moving window point of view for its convenience of description.) The insensitivity of the accelerating wakefield to the dynamic beam development is a consequence of the beam being narrow when compared to the radius at which the surface layer is located. For most of the plasma oscillation, all of the plasma electrons are outside of the beam so that from Gauss law the electrostatic field affecting them is independent of the radius of the charge inside. Thus the betatron pinching of the beam has little effect on the plasma electrons and hence the wake. The slower evolution in the front of the beam does not have any significant effect either since the slight variations in the initial trajectories of electrons become insignificant after the blown out electrons reach the surface layer. The surface layer is shown in Fig. 2, where a radial lineout of the plasma charge density at the center of the beam is plotted after $1.4 \mathrm{~m}$ of propagation. The plasma blowout as well as the surface layer are clearly visible.

Because of the invariance of the accelerating field, the expected energy gain can be predicted with confidence for a specified beam charge and profile. The longitudinal momentum $p_{z}(\cong \gamma)$ versus $c t$ phase space is shown in Fig. 3 to illustrate the expected acceleration of the beam

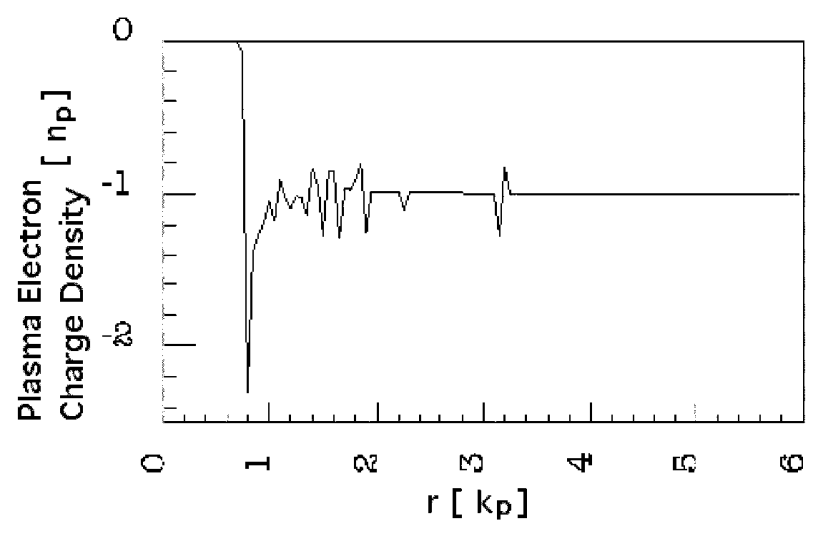

FIG. 2. A radial lineout of the background plasma electron charge density at the center of the beam after $1.4 \mathrm{~m}$ of propagation.

after $1.4 \mathrm{~m}$ of propagation. The mean, maximum, and minimum energy of the beam are plotted in $0.12 \mathrm{psec}$ bins along the length of the beam [Fig. 3(a)]. This is done in Fig. 3(a) for the actual simulation particle data after $1.4 \mathrm{~m}$. Figure 3(b), by contrast, was generated by using the initial particle data propagated for $1.4 \mathrm{~m}$ using the initial fields at the initial positions of the particles. This makes the assumption of a nonevolving field and neglects the betatron oscillation of each particle. The mean, maximum, and minimum energies resulting from these two graphs are very similar for most of the beam. The results differ only at the very end of the beam where Fig. 3(b) shows larger average and maximum energies and lower minimum energies than Fig. 3(a). The similarity between the two figures for the main part of the beam is consistent with our assumption of nonevolving wakefield if the accelerating field has a constant value within the radial range of the betatron oscillation for each particle. The differences in the tail are due to the fact that the particles in the tail at larger radii do not experience a constant accelerating field during their radial motion. For the full simulation this leads to an averaging out of the different accelerations experienced by each particle due to its transverse motion. For the particles accelerated with the initial field this averaging does not happen and the maximum and minimum energies in the beam tail of Fig. 3(b) are therefore a measure of the maximum and minimum accelerating field in that part of the wake. Based on these figures we can say that the maximum field is about $0.85 \mathrm{GeV} / \mathrm{m}$ but that the maximum energy gain by a particle after $1.4 \mathrm{~m}$ will be about $1 \mathrm{GeV}$. The maximum mean energy for a $0.12 \mathrm{psec}$ bin is $550 \mathrm{MeV}$ with about $7 \times 10^{7}$ electrons in this maximum energy bin. This is again consistent with the information in the video for these numbers. The conclusion from Figs. 3(a) and 3(b) is that the betatron oscillations do not have a significant influence on the acceleration of the beam. 


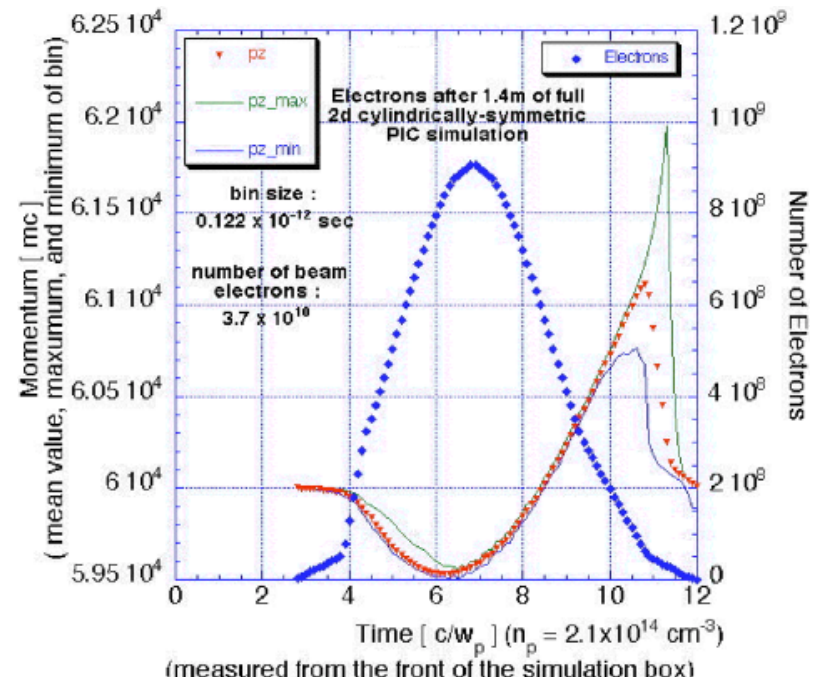

(a)

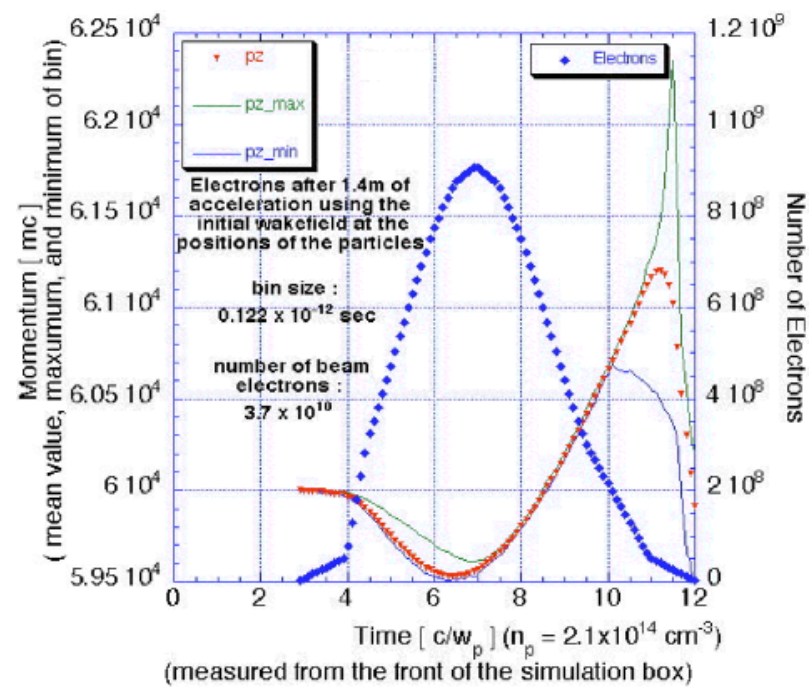

(b)

FIG. 3. (Color) The mean, maximum, and minimum energy as well as the number of electrons for each $0.12 \mathrm{psec}$ bin after $1.4 \mathrm{~m}$ of propagation using (a) the full PIC simulation and (b) the initial fields at the initial positions of the particles to propagate the beam

\section{CONCLUSIONS}

The main result of the analysis of the beam and wakefield dynamics is that the wakefield is rather insensitive to the betatron oscillation dynamics of the beam and therefore essentially constant over time. In addition, the acceleration and deceleration of the beam electrons is also not affected by the betatron oscillation either. The blowout regime therefore provides a stable and robust plasma wakefield acceleration. Energy gains on the order of $\mathrm{GeV}$ should be achievable in this blowout regime if the physical parameters of the simulations can be realized in an experiment. Far higher gradients and energy gains may be possible with shorter bunches and longer denser plasmas [3]. Such beams would undergo hundreds rather than a few betatron oscillations. Although it may be desirable to match the beam emittance to the plasma focusing strength to avoid betatron oscillations as discussed in Ref. [3], the analysis here suggests that the presence of the oscillation is not necessarily detrimental. A major issue on the scaling of scaling plasma wakefield acceleration to the 10 and $100 \mathrm{~s}$ of $\mathrm{GeV}$ is the possibility of a hosing instability of the beam [9], which might reduce the achievable energy gain and lead to emittance growth of beam. Hosing is inherently a 3D instability and is therefore absent in these $2 \mathrm{D}$ simulations. We are currently carrying out 3D simulations to study the importance of hosing and other 3D effects.

\section{ACKNOWLEDGMENTS}

We acknowledge useful conversations with the entire E-157 Collaborative Team. This work is supported by DOE Contracts No. DE-FG03-92ER40727, No. DEFG03-98DP00211, and No. DE-FG03-92ER40745, NSF Grant No. DMS-9722121, and LLNL Contract No. W7405-ENG-48.

[1] P. Chen, J. M. Dawson, R. W. Huff, and T. Katsouleas, Phys. Rev. Lett. 54, 693 (1985).

[2] E. Esarey, P. Sprangle, J. Krall, and A. Ting, IEEE Trans. Plasma Sci. 24, 252 (1996).

[3] R. Assmann et al., Stanford Linear Accelerator Center Proposal, 1997.

[4] J. B. Rosenzweig, B. Breizman, T. Katsouleas, and J. J. Su, Phys. Rev. A 44, R6189-R6192 (1991).

[5] S. Lee, T. Katsouleas, R. G. Hemker, and W. B. Mori, Phys. Rev. E 61, 7014 (2000).

[6] R. G. Hemker, F. S. Tsung, V. K. Decyk, W. B. Mori, S. Lee, and T. Katsouleas, in Proceedings of the 1999 Particle Accelerator Conference, New York (IEEE, Piscataway, NJ, 1999).

[7] J. Villasenor, Comput. Phys. Commun. 69, 306-316 (1992).

[8] K. C. Tzeng, W. B. Mori, and C.D. Decker, Phys. Rev. Lett. 76, 3332 (1996).

[9] D. H. Whittum, W. M. Sharp, S.S. Yu, M. Lampe, and G. Joyce, Phys. Rev. Lett. 67, 991 (1991). 